\title{
Fracture characterization from near-offset VSP inversion ${ }^{\top}$
}

Steve Horne, ${ }^{2,3}$ Colin MacBeth, ${ }^{2}$ J.Queen, ${ }^{4}$ W. Rizer ${ }^{4}$ and V. Cox ${ }^{4}$

\begin{abstract}
A global optimization method incorporating a ray-tracing scheme is used to invert observations of shear-wave splitting from two near-offset VSPs recorded at the Conoco Borehole Test Facility, Kay County, Oklahoma. Inversion results suggest that the seismic anisotropy is due to a non-vertical fracture system. This interpretation is constrained by the VSP acquisition geometry for which two sources are employed along near diametrically opposite azimuths about the well heads. A correlation is noted between the time-delay variations between the fast and slow split shear waves and the sandstone formations.
\end{abstract}

\section{Introduction}

Wave propagation in anisotropic media is considerably more complicated than for the isotropic case. Perhaps the most fundamental complication arises from the existence of three distinct body waves propagating with different velocities and polarizations. These form an orthogonally polarized set which is not generally coincident with the dynamic axes and, except for symmetry directions, cannot be described in terms of $\mathrm{P}, \mathrm{SH}$ and SV wavetypes. These three body waves are generally referred to as the $q P$ (quasi-compressional), $q S 1$ and $q S 2$ (fast and slow quasi-shear waves) modes. The existence of these two quasi-shear waves travelling with distinct polarizations and velocities is often referred to as shear-wave birefringence or shearwave splitting. This phenomenon has been extensively investigated and is of particular interest to petroleum geoscientists since shear-wave splitting may be interpreted in terms of aligned fracture systems (e.g. Crampin and Love11 1991; Mueller 1992). Such information may be critically important to the successful development of hydrocarbon reservoirs whose permeability is controlled by fracturing (Ehlig-Economides, Ebbs and Meehan 1990; Ata and Michelena 1995). A method is therefore desirable which may be applied to invert these measurements

${ }^{1}$ Received October 1995, revision accepted January 1996.

2 British Geological Survey, Murchison House, West Mains Road, Edinburgh, EH9 3LA, Scotland, UK.

3 Department of Geology and Geophysics, Grant Institute, University of Edinburgh, West Mains Road, Edinburgh, EH9 3JW, Scotland, UK.

4 Conoco Inc., Ponca City, OK 74603, USA. 
of shear-wave splitting. Unfortunately, the formulation of the inverse problem is impeded by the complicated nature of wave propagation in anisotropic media (Helbig 1994). We show how a global optimization method may be applied to this problem.

\section{Genetic algorithms}

Genetic algorithms (GA) are a class of search methods based on an analogy with natural evolution (Goldberg 1989). A GA uses a collection of models, analogous to a population, which are individually represented in a coded form, analogous to a chromosome. These are then manipulated by 'genetic operators' in such a way that better models, on average, are generated. Unfortunately, the underlying search mechanisms explaining the successful behaviour of GAs have yet to be clearly identified (Fogel and Stayton 1994). None the less, these methods have generally been found to be useful in many diverse fields (Davis 1991), including the geophysics industry (e.g. Sen and Stoffa 1992).

Global optimization schemes, such as GAs or simulated annealing, typically sample several thousands of models during a search so that any forward-modelling scheme requiring more than a few seconds becomes prohibitively expensive in computational time. We therefore require a forward-modelling scheme which is both accurate and efficient. The most suitable approach is offered by a ray-tracing scheme.

\section{Inversion requirements}

The ray-tracing method is a high-frequency approximation which effectively follows the advance of a plane wavefront through a structure by application of Snell's law. This statement is true for wave propagation in both isotropic and anisotropic media although Snell's law needs some reformulation for the anisotropic case (Helbig 1994). Ray tracing in continuous anisotropic media essentially requires the integration of six coupled differential equations (Červený 1972). These may be solved in a variety of ways for example, using Runge-Kutta methods (Li, Leary and Akiki 1990) or finite-difference methods (Pereya, Lee and Keller 1980). Although raytracing schemes are fast, efficient, and may be applied to complex heterogeneous 3D structures, considerable errors may be introduced for singular regions (Gajewski and Pšenčik 1990). It should also be remembered that ray tracing implicitly assumes plane wavefronts so that the calculations are for the phase rather than the group velocities.

The ray-tracing method is a very general technique which may be applied to complicated 3D heterogeneous anisotropic models. There will clearly be a trade-off between the computational speed and the model complexity. It is therefore possible to decrease the computational time significantly by increasing the model simplicity. For this reason we approximate the models to a 1D stack of plane layers. A further increase in speed can be achieved if out-of-sagittal-plane deviations are neglected. This reduces the ray-tracing method to a root-searching problem as a function of one

C 1 (x) 1:uropean Association of Geoscientists \& Engineers, Geophysical Prospecting, 45, 141-164 
variable. This second approximation will be valid in the case of weak anisotropy or in the case that the propagation plane is a plane of symmetry.

\section{Modelling procedure}

The concept that underlies ray tracing in both isotropic and anisotropic media is the conservation of the tangential components of slowness as described by Snell's law. In the case of a horizontally plane layered model, this implies that the horizontal components, $p_{x}$ and $p_{y}$, are the quantities conserved at the interfaces. In the isotropic case the vertical component of slowness is simply given by $p_{z}^{2}=\left(1 / v_{\text {iso }}^{2}-p_{x}^{2}-p_{y}^{2}\right)$. However, for the anisotropic case, the velocity is a function of the propagation direction which complicates matters considerably. In the case of triclinic symmetry, the vertical component of slowness, $p_{z}$, can be derived by the solution of a sextic equation. The use of this equation is to be avoided because of the numerical imprecision associated with round-off errors and the complexity of the coefficients (Fryer and Frazer 1987). However, a considerable simplification is achieved if the medium possesses a monoclinic or a higher symmetry with a horizontal plane of symmetry. In this case the slowness may be written in terms of a cubic, the three roots of which are simply the squares of the vertical slowness, p,

The approach that we use is initially to construct a table of slowness curves before implementing the ray-tracing algorithm. Intermediate values of slowness can be derived by the application of interpolation methods. This approach has the advantage that a horizontal plane of symmetry need not be assumed, as is the case with the analytical slowness equations, but it should be noted that a loss of precision may be involved with the interpolation approach. Horizontal and vertical slownesses are calculated for a range of angles of incidence between vertical and horizontal directions in the sagittal plane by solution of the Kelvin-Christoffel equation. Care must be taken in the choice of angles of incidence used in the interpolation scheme, since the derivatives of the slowness curve will be sensitive to the horizontal slowness sampling interval. For example, if the slowness curve is sampled with equispaced angles then the errors in the derivatives will increase towards the horizontal propagation directions. A more appropriate scheme is to calculate the incremental change in the angle of incidence so that the horizontal slowness is sampled at approximately equal intervals.

These interpolation tables are used in the ray-tracing algorithm to calculate the vertical slowness component of a transmitted wave for a given horizontal slowness. The horizontal and vertical slownesses define the propagation direction within the layer. In this way, the ray may be traced through the stack of layers for a trial horizontal slowness value. The ray is terminated after it has travelled a horizontal distance corresponding to the source-geophone distance. The ray-tracing algorithm therefore searches over the horizontal slowness parameter for a ray connecting the source and geophone. This is achieved using a shooting method, whereby an initial course scan is performed over the horizontal slowness to bracket the solution. The 
horizontal slowness root is then refined using standard numerical procedures (Press et al. 1992).

Care must be taken when applying Snell's law at each interface to ensure that there is continuity between the incident and transmitted shear waves. This implies that the body waves must be separated according to the polarization of the wavetype. For hexagonal materials this may be achieved as one of the shear waves is entirely independent of the two other wavetypes (Musgrave 1970) so that it is possible to distinguish the two shear waves by application of the equation

$$
\rho V_{q S}^{2}-c_{44}\left(1-q^{2}\right)-c_{66} q^{2}=0
$$

where the symmetry axis is aligned along the $x_{1}$-direction, $\rho$ is the density, $V_{q S}$ is the velocity of the shear wave polarized in the plane perpendicular to the symmetry axis, 4 is the direction cosine in the $x_{1}$-direction and $c_{44}$ and $c_{66}$ are elastic constants.

\section{Inversion procedure}

We use the GA to minimize a least-squares misfit function defined as

$$
f\left(\mathbf{m}, \tau^{0}, \mathbf{p}^{0}\right) . \quad \frac{1}{2}\left[\Delta \tau\left(\tau^{0}, \tau^{\mathrm{m}}, \delta \boldsymbol{\tau}\right) . \quad \Delta p\left(\mathbf{p}^{0} \mathbf{p}^{\mathrm{m}}, \delta \mathbf{p}\right)\right]
$$

where

$$
\Delta \tau\left(\boldsymbol{\tau}^{0}, \boldsymbol{\tau}^{\mathrm{m}}, \delta \boldsymbol{\tau}=\frac{1}{N} \sum_{j=1}^{N}\left(\frac{\tau_{j}^{0}-\tau_{j}^{\mathrm{m}}}{\delta \tau_{j}}\right)^{2}\right.
$$

and

$$
\Delta p\left(\mathbf{p}^{0}, \mathbf{p}^{\mathrm{m}}, \delta \mathbf{p}\right)=\frac{1}{N} \sum_{j=1}^{N}\left(\frac{p_{j}^{0}-p_{j}^{\mathrm{m}}}{\delta p_{j}}\right)
$$

$\tau$ and $\mathrm{p}$ are vectors of the time delays and the $q S 1$ polarizations in the horizontal plane. $\mathrm{m}$ is the model parameter vector describing the anisotropic perturbation to be added to the isotropic layered model for which shear-wave splitting observations are calculated by the ray-tracing algorithm. We describe results obtained using a model vector defined in terms of crack parameters. The subscript $j$ identifies the observation number and $\mathrm{N}$ is the total number of observations. The superscripts identify quantities relating to the model, superscript $\mathrm{m}$, and the observed, superscript 0 , vectors. $\delta \tau$ and $\delta \mathbf{p}$ are error estimates associated with the observed time delays, $\tau^{0}$, and $q S 1$ polarizations, $\mathbf{p}^{0}$, respectively. The shear-wave splitting estimates are assigned estimated errors of 10 " for the $q S 1$ polarizations and $2 \mathrm{~ms}$ for the time delays. $q S 1$ polarization estimates associated with time delays less than $2 \mathrm{~ms}$ are assigned an estimated error of 20". This reflects the uncertainty in the shear-wave estimation techniques for which the $q S 1$ polarizations are unlikely to be well 
resolved. This choice of objective function implies that the errors follow a Gaussian distribution.

The predicted shear-wave splitting observations, $\tau^{\mathrm{m}}$ and $\mathbf{p}^{\mathrm{m}}$, are calculated for a layered anisotropic model based on a perturbation defined by the model vector, $\mathrm{m}$. The model for the Conoco Borehole Test Facility (CBTF) uses 18 layers whose velocities, densities and thicknesses are measured from logging information and, for shallow depths, reverse VSP surveys. This layered model is then split into four different anisotropic zones corresponding to the observed discontinuities in the shear-wave splitting estimates from the two VSP surveys (Fig. 1). The imposition of a common symmetry axis for each of these regions is applied to avoid any problems associated with multiply split shear waves. Furthermore, inversions are only attempted for hexagonal symmetry systems due to limitations within the implemented ray-tracing scheme.

The model space is parametrized using equivalent media formulations based upon Hudson's crack formulations (Hudson 1980) with each anisotropic zone described by three parameters. These are the crack density (CD), the aspect ratio (AR) and the crack content (CT). A logarithmic function is used to represent the aspect ratio parameter since seismic data from near-offset VSPs are relatively insensitive to this parameter (MacBeth 1991). The GA control parameters for the three different inversions are given in Table 1 . We also use several other 'advanced' operations which are described by Goldberg (1989) and Horne (1995). Other parametrization schemes based on a perturbation from the isotropic tensor and the Thomsen parameters have also been attempted but are not reported here as they yield similar results (Horne 1995).
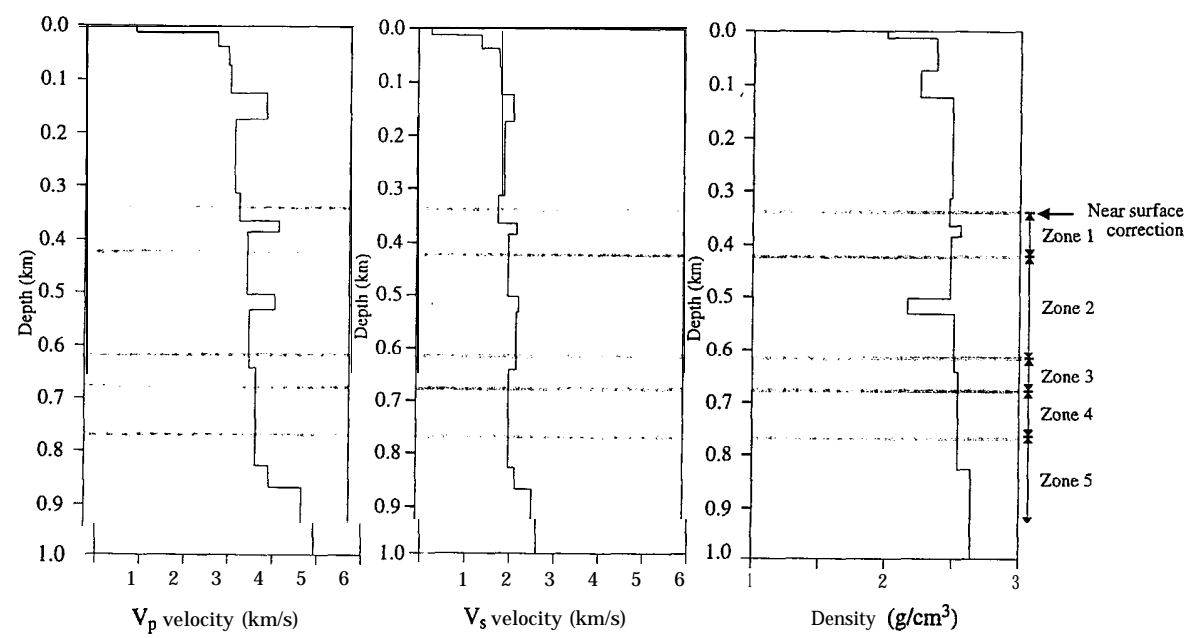

Figure 1. Velocity and density model used in the inversion scheme. Five anisotropic zones are identified from the shear-wave estimation results as indicated. Also indicated is the depth at which the near-surface correction is applied to both VSP data sets. 
Table 1. GA inversion parameters.

\begin{tabular}{lcr}
\hline \multicolumn{2}{c}{ GA Parameter } & \\
\hline Population size & $\mathrm{N}_{\text {pop }}$ & 60 \\
Number of generations & $\mathrm{N}_{\text {gen }}$ & 60 \\
Crossover probability & $\mathrm{PC}$ & 0.950 \\
Mutation probability & $p_{\mathrm{m}}$ & 0.010 \\
Sharing & & $\sqrt{ }$ \\
Reordering ('Inversion') & & $\sqrt{ }$ \\
Hill climbing ('G-bit improvement') & & $\sqrt{ }$ \\
\hline
\end{tabular}

\section{Application to field data}

The ray-tracing algorithm is incorporated into the GA which we then apply to inverting shear-wave splitting observations from two similar near-offset VSP experiments. The near-offset VSP experiments were conducted at two different wells within $1 \mathrm{~km}$ of each other at the CBTF. The site is located in north central Oklahoma in a relatively simple structural setting. The section penetrated by the wells consists of relatively flat-lying Permian and Pennsylvanian shales, limestones, and sandstones, as described by Queen and Rizer (1990).

\section{Near-offset VSP acquisition geometries}

We identify the two near-offset VSPs as the 33-1 VSP and the Peel VSP, referring to the two wells about which the experiments were shot. The Peel well is located approximately $740 \mathrm{~m}$ to the south-east of well 33-1 (Fig. 2). The acquisition geometries for these two experiments are similar in terms of the angles of incidence sampled, but the azimuthal propagation directions sampled correspond to almost diametrically opposite raypaths. The importance of information obtained along such opposite azimuths will become apparent and will be discussed in relation to vertical and non-vertical crack systems. A summary of the acquisition geometries and the source and receiver parameters is given in Tables 2 and 3. Plan views of both VSP acquisition geometries are shown in Fig. 3.

\section{$33-l \quad V S P$}

Vibroseis sources were located $36 \mathrm{~m}(120 \mathrm{ft})$ along the azimuth $\mathrm{N} 279^{\circ} \mathrm{E}$ relative to well 33-1. The Vibroseis sources were operated in in-line, cross-line and vertical directions. Seismograms were recorded at 50 levels between depths of $152 \mathrm{~m}(500 \mathrm{ft})$ and $887 \mathrm{~m}(2910 \mathrm{ft})$ using a constant geophone spacing of $15 \mathrm{~m}(50 \mathrm{ft})$. The horizontally polarized Vibroseis sources were swept through a frequency range of 6 to $51 \mathrm{~Hz}$, whereas the vertically polarized vibrator was swept through a range of 12 to $102 \mathrm{~Hz}$. 
$\oplus$
$33-1$
(N $68^{\circ} 0631$ E 229 2716$)$

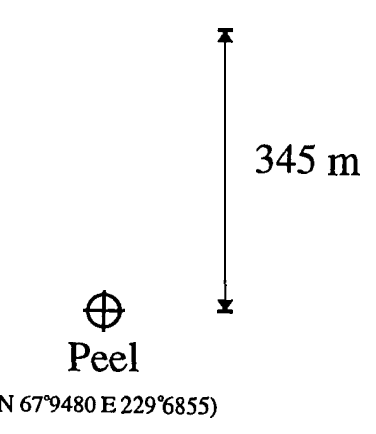

$w-\int_{s}^{N}=$

$640 \mathrm{~m}$

Figure 2. Plan view of the Conoco Borehole Test Facility, Kay County, Oklahoma showing the relative location of the 33-1 and Peel wells. Grid references are USGS state plane coordinates.

Table 2. Data acquistion parameters for the 33-1 VSP.

\begin{tabular}{lc} 
Sources & \\
\hline Horizontal vibrators x 2 & $51-6 \mathrm{~Hz}$ \\
Sweep & $279 "$ \\
Azimuth & $36 \mathrm{~m}, 30 \mathrm{~m}$ \\
Offsets & $102-12 \mathrm{~Hz}$ \\
Vertical vibrator & $279 "$ \\
Sweep & $39 \mathrm{~m}$ \\
Azimuth & $30 \mathrm{~s}$ \\
Offset & $32 \mathrm{~s}$ \\
Sweep length & $0.5 \mathrm{~s}$ \\
Record length & $2 \mathrm{~m} \mathrm{~s}$ \\
Taper & \\
Sample interval & \\
Downhole Sonde & \\
\hline Desco 3-C receiver & \\
Depth locations & \\
\hline 50 levels, 152 m through to $887 \mathrm{~m}$ at $15 \mathrm{~m}$ intervals \\
\hline
\end{tabular}


Table 3. Data acquisition parameters for the Peel VSP.

\begin{tabular}{lc} 
Sources & \\
\hline Horizontal vibrators x 2 & \\
Sweep & $7-60 \mathrm{~Hz}$ \\
Azimuth & $122^{\prime}$ \\
Offsets & $65 \mathrm{~m}$ \\
Vertical vibrator & $7-15 \mathrm{~Hz}$ \\
Sweep & $123 "$ \\
Azimuth & $71 \mathrm{~m}$ \\
Offset & $14 \mathrm{~s}$ \\
Sweep length & $15 \mathrm{~s}$ \\
Record length & $0.2 \mathrm{~s}$ \\
Taper & $1 \mathrm{~m} \mathrm{~s}$ \\
Sample interval & \\
Downhole Sonde & \\
\hline
\end{tabular}

\section{GSC-20D 3-C receiver}

Depth locations

44 levels, $306 \mathrm{~m}$ through to $951 \mathrm{~m}$ at $15 \mathrm{~m}$ intervals

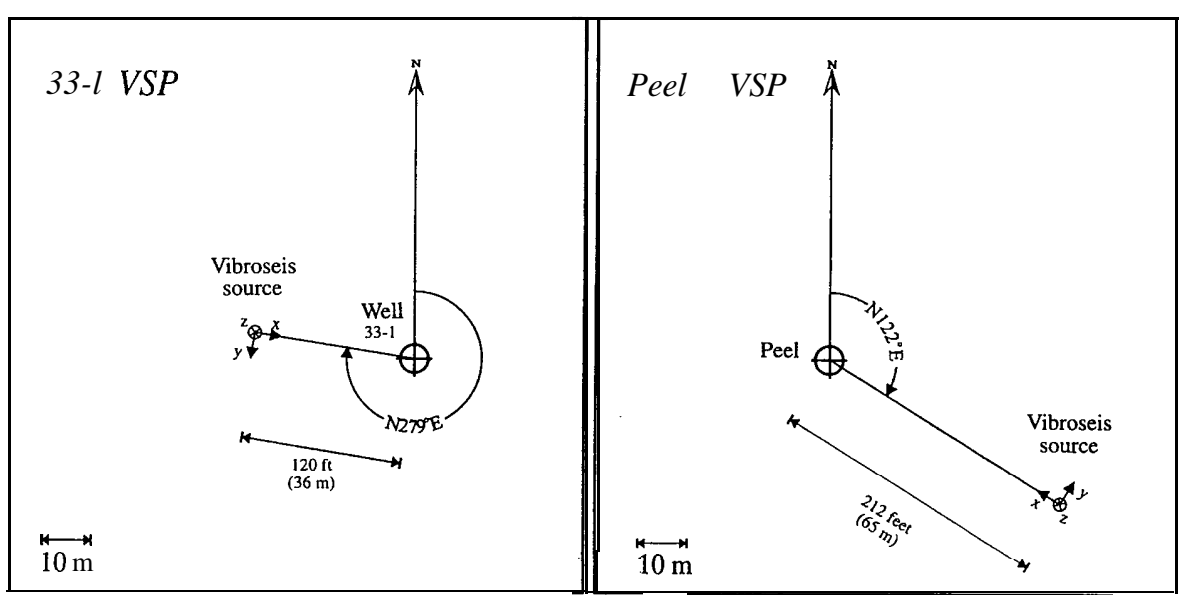

Figure 3. Plan views showing the acquisition geometries used to obtain the multicomponent near-offset VSP data sets.

(C) 1997 European Association of Geoscientists \& Engineers, Geophysical Prospecting, 45, 141- 164 


\section{Peel VSP}

In this experiment the Vibroseis source trucks were sited $6.5 \mathrm{~m}(210 \mathrm{ft})$ from the Peel well along the azimuth $\mathrm{N} 122^{\circ} \mathrm{E}$. In-line, cross-line and vertical source orientations were used to excite seismic energy which was recorded at 44 equispaced depth levels between $306 \mathrm{~m}(1003 \mathrm{ft})$ and $951 \mathrm{~m}$ (3 $120 \mathrm{ft})$. The horizontally polarized vibrators were downswept from 60 to $7 \mathrm{~Hz}$, and the vertically polarized vibrator downswept from 150 to $7 \mathrm{~Hz}$.

\section{Processing sequence applied to shear-wave data}

We apply a common processing sequence to both VSP data sets for the purposes of consistency. The data processing steps that we use are:

1. Horizontal geophone rotation into the in-line and cross-line directions. This is necessary to correct for downhole tool spin.

2. f-k filtering, to separate the up- and down-going shear wavefields.

3. Near-surface correction, to remove any possible inconsistencies due to variations in the near-surface layers (MacBeth et al. 1995).

After each of the processing steps above a visual check is made of the processed seismograms and automatic techniques are applied to extract shear-wave splitting information. The purpose of this is to verify that these processing steps do not distort the shear wavefield.

\section{$33-l \quad V S P$}

The shear-wave splitting is quantified in terms of the fast shear-wave polarization and the time delay between the split shear waves by the use of an analytical implementation of the Alford rotation (Zeng and MacBeth 1993). Estimation results for the 33-1 VSP data after each of the processing steps are shown in Fig. 4. The polarization estimates show little change after application of $f-k$ filtering and nearsurface correction and indicate a constant shear-wave polarization of approximately $\mathrm{N} 50^{\circ} \mathrm{E}$. This result disagrees with that reported by Queen et al. (paper presented at 5th international workshop on seismic anisotropy, Banff, Canada, 1992) for which the fast shear-wave polarization was found to be $\mathrm{N} 75^{\circ} \mathrm{E}$. This discrepancy may arise from a polarity confusion which cannot, at present, be resolved on the basis of observer logs. The time-delay estimates after near-surface correction are shifted by approximately $5 \mathrm{~ms}$ so that, as expected, there is negligible splitting at the depth at which the correction is applied. The rate of increase of the time delay with depth is not continuous and significant discontinuities can be identified at the four depth levels, $422 \mathrm{~m}, 617 \mathrm{~m}, 677 \mathrm{~m}$ and $767 \mathrm{~m}$, as indicated on Fig. 4. The section between the near-surface correction reference level and the first discontinuity at $422 \mathrm{~m}$ reveals a moderate increase in the time delay of approximately $12 \mathrm{~ms} / \mathrm{km}$. The second anisotropic zone lying between $422 \mathrm{~m}$ and $617 \mathrm{~m}$ corresponds to a time-delay 

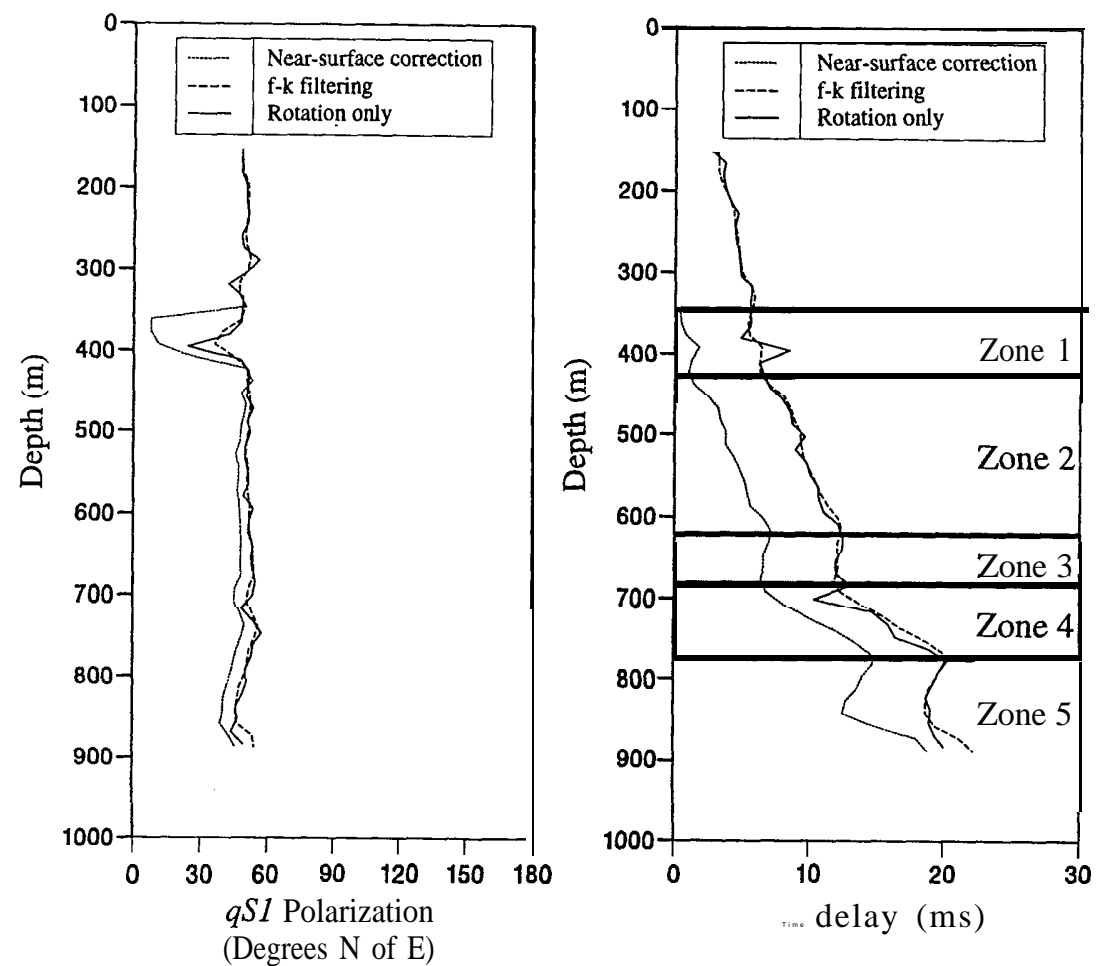

Figure 4. Shear-wave estimation results for the 33-1 VSP at various processing stages.

gradient of $33 \mathrm{~ms} / \mathrm{km}$. The third zone is apparently isotropic as there is no appreciable change in the time-delay estimates over this depth range. Between $677 \mathrm{~m}$ and $767 \mathrm{~m}$ there is a significant increase in the time-delay gradient to $97 \mathrm{~ms} / \mathrm{km}$. Below this depth range, the time-delay estimates decrease to a minima of $18 \mathrm{~ms}$ at $827 \mathrm{~m}$ and then increase to $23 \mathrm{~ms}$ for the deepest recording at $887 \mathrm{~m}$.

\section{Peel VSP}

Results from the application of shear-wave estimation techniques are shown in Fig. 5. Unlike the results for the 33-1 VSP, the near-surface correction has a considerable effect in clarifying the estimation results. Before near-surface correction the estimated $q S 1$ polarizations decrease steadily with depth from $\mathrm{N} 90^{\circ} \mathrm{E}$ at shallow levels to $\mathrm{N} 60^{\circ} \mathrm{E}$. After the correction this rotation becomes less apparent and the $q S 1$ polarizations are shifted towards $\mathrm{N} 55^{\circ} \mathrm{E}$. Before the near-surface correction there is no clear time-delay variation with depth as observed for the 33-1 VSP estimation results. None the less, possible discontinuities can be observed at depths similar to those identified in the 33-1 VSP results. However, after the near-surface correction the time-delay gradients can be measured with more confidence. The first anisotropic 

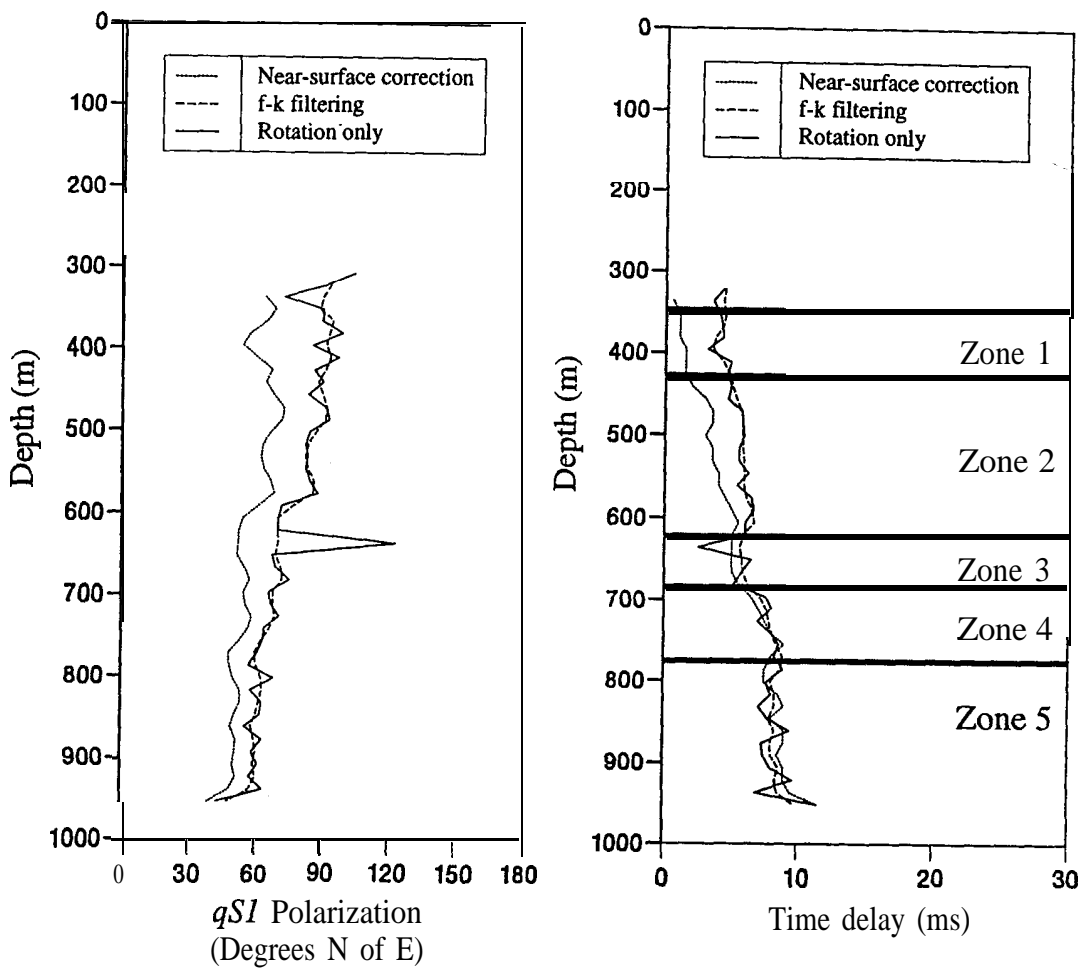

Figure 5. Shear-wave estimation results for the Peel VSP at various processing stages.

zone, between the reference level at which the near-surface correction is applied and $422 \mathrm{~m}$ depth, corresponds to a time-delay gradient of $11 \mathrm{~ms} / \mathrm{km}$. This increases to approximately $20 \mathrm{~ms} / \mathrm{km}$ in the second zone which extends to a depth of $617 \mathrm{~m}$. No significant changes in the time delays are observed between $617 \mathrm{~m}$ to $677 \mathrm{~m}$ so that this zone is likely to be isotropic. A time-delay gradient of $42 \mathrm{~ms} / \mathrm{km}$ is observed between $677 \mathrm{~m}$ and $766 \mathrm{~m}$. Below this depth there is no clear trend in the time delays and this zone appears isotropic.

A comparison of the estimation results from the two VSPs reveals a similar timedelay variation with discontinuities in the time-delay gradients occurring at similar depths (Figs 4 and 5). There are also similarities in the relative time-delay gradients in the five anisotropic regions with the results obtained from the 33-1 VSP. In particular, shear-wave splitting is pronounced in the second and fourth regions with the intermediate region being isotropic. However, the overall degree of splitting for the Peel VSP is significantly smaller than that observed for the 33-1 VSP. An obvious explanation is offered in terms of lateral variability since the two wells are $740 \mathrm{~m}$ apart. Although this is a plausible explanation, the similarity in the variations of the time-delay estimates with depth suggests that there may be some 
correspondence between the observed anisotropy in the two wells. Furthermore, the two VSPs are shot in a relatively simple geological setting so that lateral changes are unlikely, although this possibility cannot be ruled out. The inversion results that we obtain show that such lateral changes need not be invoked to explain these shearwave splitting observations.

\section{Stratigraphic correlation with shear-wave birefringence}

The results we obtain for the observed shear-wave birefringence from both nearoffset VSPS show a clear correlation with the reported stratigraphy at the CBTF. We show this in Fig. 6, where a stratigraphic depth section of the geology at the test site is plotted on the same depth scale as the splitting estimates. The large time-delay

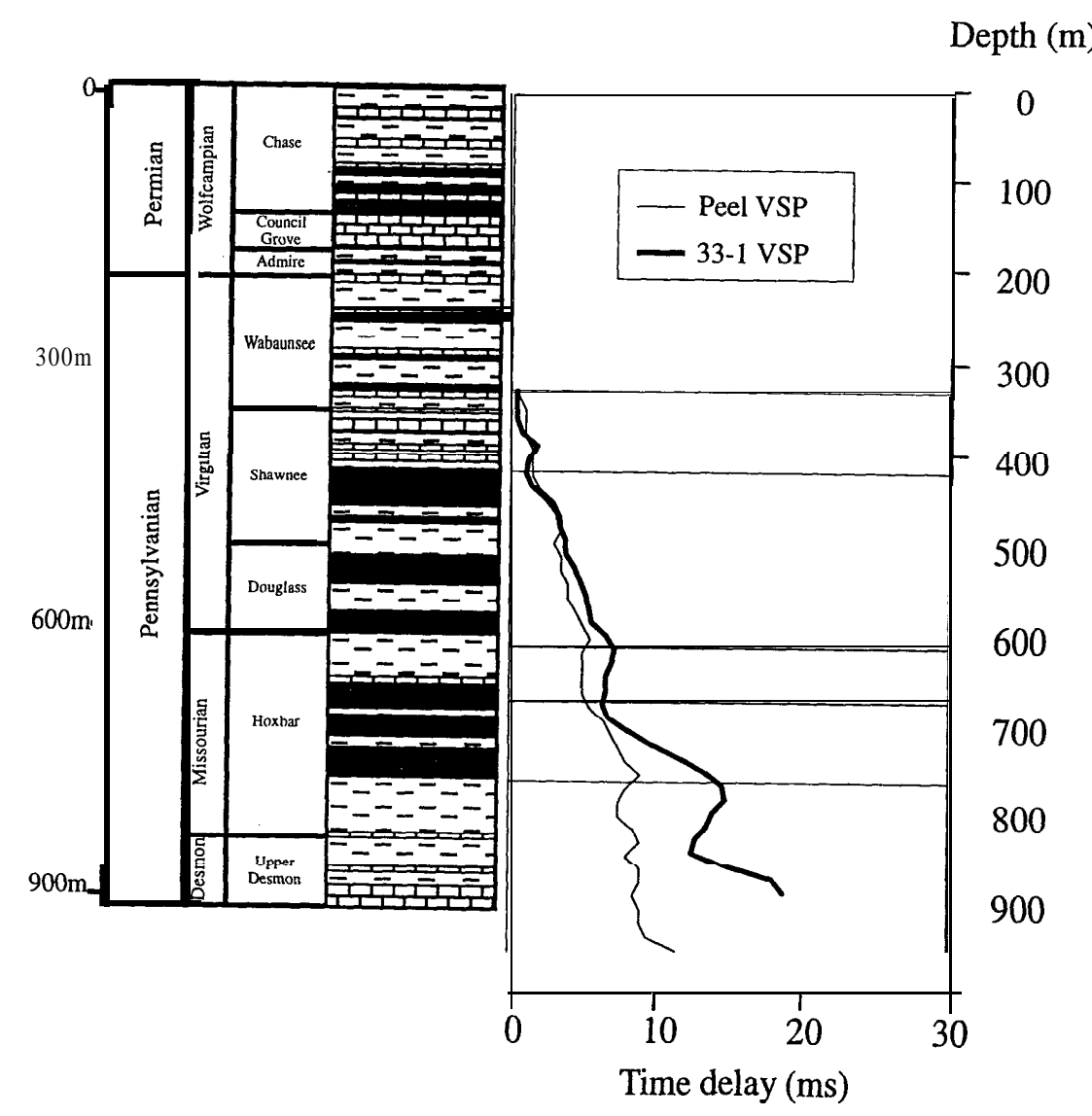

Figure 6. A comparison of the shear-wave estimation results (after application of $f-k$ filtering and near-surface correction) obtained for the 33-1 and Peel VSP data sets with the stratigraphy reported at the Conoco Borehole Test Facility (after Queen and Rizer 1990). Note the correlation between the time delays and the sandstone formations. 
gradients for the two depth ranges $422-617 \mathrm{~m}$ and $677-767 \mathrm{~m}$ correspond to formations with considerable amounts of sandstone interbedded with shale. This correlation suggests that the shear-wave birefringence is sensitive to the presence of open fractures in the sandstones. The depth interval at $700 \mathrm{~m}$ has also been observed to correspond to fluid loss in all of the deep wells at the CBTF which is attributed to large open fractures within this zone (Queen et al. 1992, as above). Alternatively, these sandstone formations may exhibit preferential grain alignment due to depositional conditions leading to equivalent anisotropic behaviour (Helbig 1994). However, the coincidence of the $q S 1$ polarizations with the maximum compressive stress direction (Zoback and Zoback 1980) suggests that the observed seismic anisotropy is not related to such depositional fabrics. Furthermore, examination of core samples from the test site do not indicate any depositional fabrics with the required orientation to explain these observations (Queen and Rizer 1990).

\section{Inversion results}

Model parameters for the best solution found by the GA are given in Table 4. The best model misfit is less than 1.0 indicating that, on average, all the model observations are within the estimated error bounds (Fig. 7). This model predicts a fracture system striking $\mathrm{N} 50^{\circ} \mathrm{E}$ and dipping 18 " from the vertical to the south-east. The predicted $q S 1$ polarizations remain constant as a function of depth and are orientated parallel to the fracture strike. This behaviour appears to model most of the observed $q S 1$ polarizations at depths below $600 \mathrm{~m}$ for the Peel VSP and below $450 \mathrm{~m}$ for the 33-1 VSP. For the 33-1 VSP, the oscillation in the polarizations above $450 \mathrm{~m}$ could not be modelled. This feature appears to be related to the poor resolution of $q S 1$ polarizations for which the associated time delay is small.

In the case of the Peel VSP, the rotation in $q S 1$ polarizations observed with depth is not reproduced. Unlike the estimates from the 33-1 VSP, this behaviour is not introduced after the near-surface correction although this processing step reduces this effect. The inability of the model observations to reproduce this behaviour could

Table 4. The best solution found using the GA for the Hudson scheme.

\begin{tabular}{lccc}
\hline & CD & AR & CT \\
\hline Zone 1 & 0.079 & $10^{-2}$ & wet \\
Zone 2 & 0.022 & $10^{-4}$ & dry \\
Zone 3 & 0.025 & $10^{-2}$ & dry \\
Zone 4 & 0.097 & $10^{-3}$ & dry \\
Zone 5 & 0.405 & $10^{-4}$ & dry \\
Crack Strike (Degrees N of E) & & $50^{\circ}$ & \\
Crack Dip (Degrees from vertical) & & $18^{\prime \prime}$ to south-east & \\
Misfit $f\left(\mathbf{m}, \tau^{0}, \mathbf{p}^{0}\right)$ & 0.265 & \\
\hline
\end{tabular}




\section{3-1 VSP}
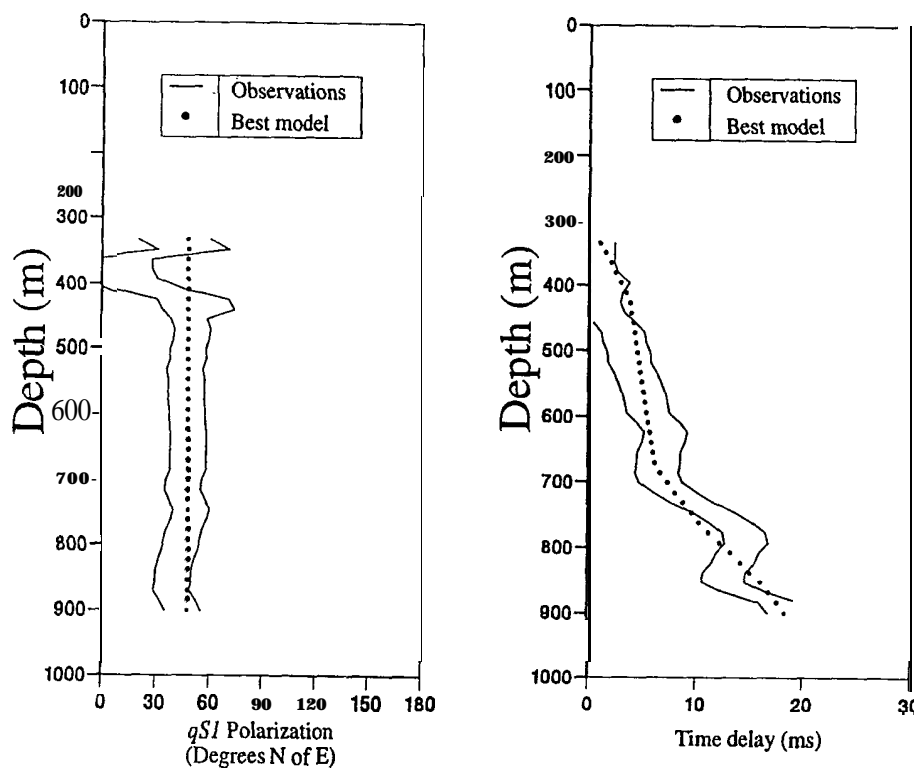

Peel VSP
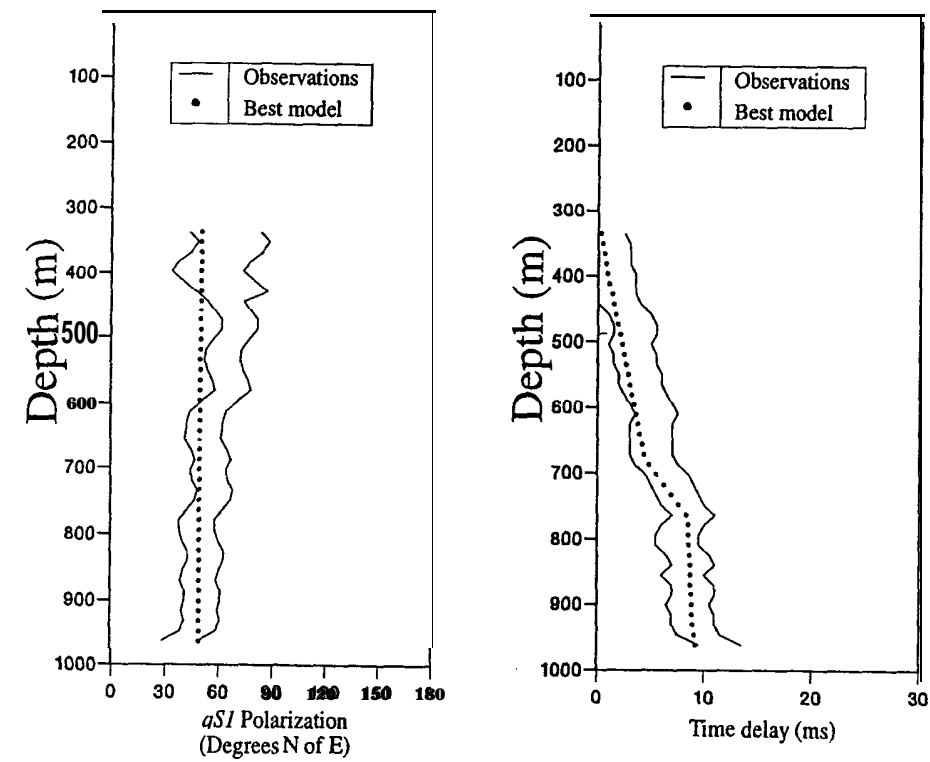

Figure 7. A comparison of the observed shear-wave splitting estimates with those predicted for the best model obtained using the GA. 
lie in the failure of the optimization method to converge or in the assumptions employed in the forward-modelling scheme. It is possible that the GA fails to converge to a lower misfit value since there is no proof that such methods are able to find the global minima. The alternative explanation of restrictive assumptions which do not allow a realistic representation of the problem is now addressed. Essentially there are three main assumptions used in the forward modelling. These are the approximation of the geology at the test site to a $1 \mathrm{D}$ stack of plane layers, the use of a ray method and the representation of the anisotropy as a transversely isotropic medium with an arbitrary orientation of the symmetry axis which is constant with depth. This first assumption is judged to be valid through the consideration of geological data from the CBTF. The second assumption of the ray-tracing method appears justified through comparison tests with full waveform synthetics generated using the reflectivity method (Horne 1995). The remaining assumption of transverse isotropy may be inappropriate since horizontal fine layering and subvertical fracturing, both of which are likely to be present at the test site, lead to equivalent media of the monoclinic symmetry class. For these systems, at near-vertical propagation directions the observed anisotropy will be dominated by the nearvertical fractures. On moving away from the vertical direction the fine layering anisotropy becomes increasingly dominant and for some systems this leads to the $q S 1$ shear wave being polarized parallel to the radial direction. This combination of subvertical fracturing and fine layering anisotropy can be used to explain the measured $q S 1$ behaviour observed for both VSPs. For the 33-1 VSP, the source is located along the azimuth $\mathrm{N} 279^{\circ} \mathrm{E}$. This implies that shear-wave energy is propagating closer to the supposed fracture orientation of $\mathrm{N} 50^{\circ} \mathrm{E}$ dipping $18^{\prime}$ to the south-east. This compares with the Peel VSP source which is located approximately downdip along azimuth $\mathrm{N} 122^{\circ} \mathrm{E}$. Thus, the $q S 1$ polarizations observed from the 33-1 VSP will be dominated by the fracturing anisotropy, whereas the $q S 1$ polarizations for the Peel VSP will be more sensitive to the fine-layering anisotropy. Equal area plots for the situations described are shown in Fig. 8. Unfortunately, a forward-modelling scheme able to produce accurate results for such monoclinic symmetry systems could not be efficiently implemented within the GA.

The majority of the predicted time delays fall within the estimated error bounds for both VSPs (Fig. 7). The poorest agreement occurs in zone 5, below $800 \mathrm{~m}$, for the 33- 1 VSP. In this zone the observed time delays decrease between $800 \mathrm{~m}$ and $850 \mathrm{~m}$ and increase below this depth. For this depth range the raypaths are essentially vertical with the straight line angles of incidence ranging between 2.4" and 2.6". Since the angular variation is essentially the same over this interval it is unlikely that this variation is due to anisotropic propagation effects. Decreases in time delay can be explained in terms of multiple splitting which may be caused by a change in the symmetry system or its orientation. Such a change can be introduced through a variation in the fracture orientation with depth. However, the implemented raytracing scheme assumes there is no such change and this effect cannot be modelled 


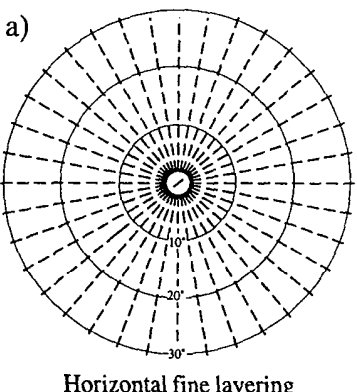

Horizontal fine layering

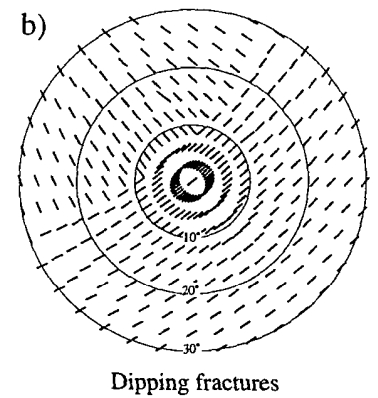

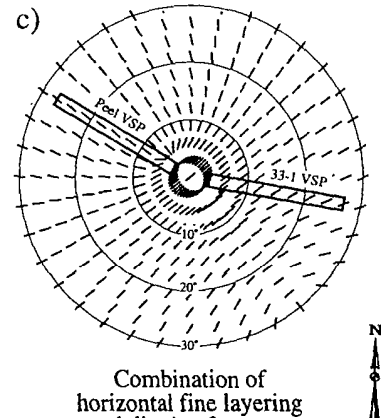

and dipping fractures

Figure 8. Lower-hemisphere equal-area plots showing the $q S 1$ polarizations between angles of incidence 0" and 30" for (a) a TIV medium constructed from horizontal fine layering, (b) a TI medium constructed from Hudson cracks dipping 24" to the south-east and striking $\mathrm{N} 50^{\circ} \mathrm{E}$. (c) A monoclinic medium constructed by combining horizontal fine layering and the dipping crack systems shown in (a) and (b). Marked on this figure are the two angular apertures corresponding to the Peel and 33-1 VSPs. For the 33-1 VSP, the $q S 1$ polarizations are aligned with the crack strike, whereas the polarizations measured using an aperture corresponding to that used for the Peel VSP show a rotation towards the radial direction with increasing incidence.

with the present inversion scheme. It should be emphasized that this assumption is necessary to construct an efficient forward-modelling scheme and is not a limitation of either ray tracing or the GA optimization scheme.

\section{Non-uniqueness}

The acceptable models, $f\left(\mathbf{m}, \tau^{0}, \mathbf{p}^{0}\right)<1.0$, sampled by the GA are shown in distancemisfit scatter plots (Fig. 9). For these plots, the multiparameter model vector is represented in terms of a single parameter, the distance $\mathrm{D}(\mathrm{m})$, which is essentially a normalized form of the vector magnitude. These plots allow a convenient visualization of the sampling distribution of high-dimension parameter spaces. The problem is clearly non-unique and within this window of acceptable solutions there are 233 models.

Of particular interest is the non-uniqueness associated with the crack orientation. We show this by plotting the strike and dip for each of the acceptable models using a scatter plot for which each dot is shaded according to that model's misfit value (Fig. 10). The models are clustered about the best model between crack strikes of $\mathrm{N} 35^{\circ} \mathrm{E}$ and $\mathrm{N} 65^{\circ} \mathrm{E}$ and crack dips between $10^{\prime \prime}$ and 40 " to the south-east. The fracture dips for the better models are concentrated about values of approximately 20 " to the south-east. This clustering is controlled by the time-delay observations. The reason for this can be clearly explained using Fig. 11, in which velocity sheets and lowerhemisphere equal-area plots of time delays are shown for equivalent anisotropic media constructed from vertical fractures and subvertical fractures. Referring to the cross-sections of both the velocity sheets and the time-delay plots in the plane 


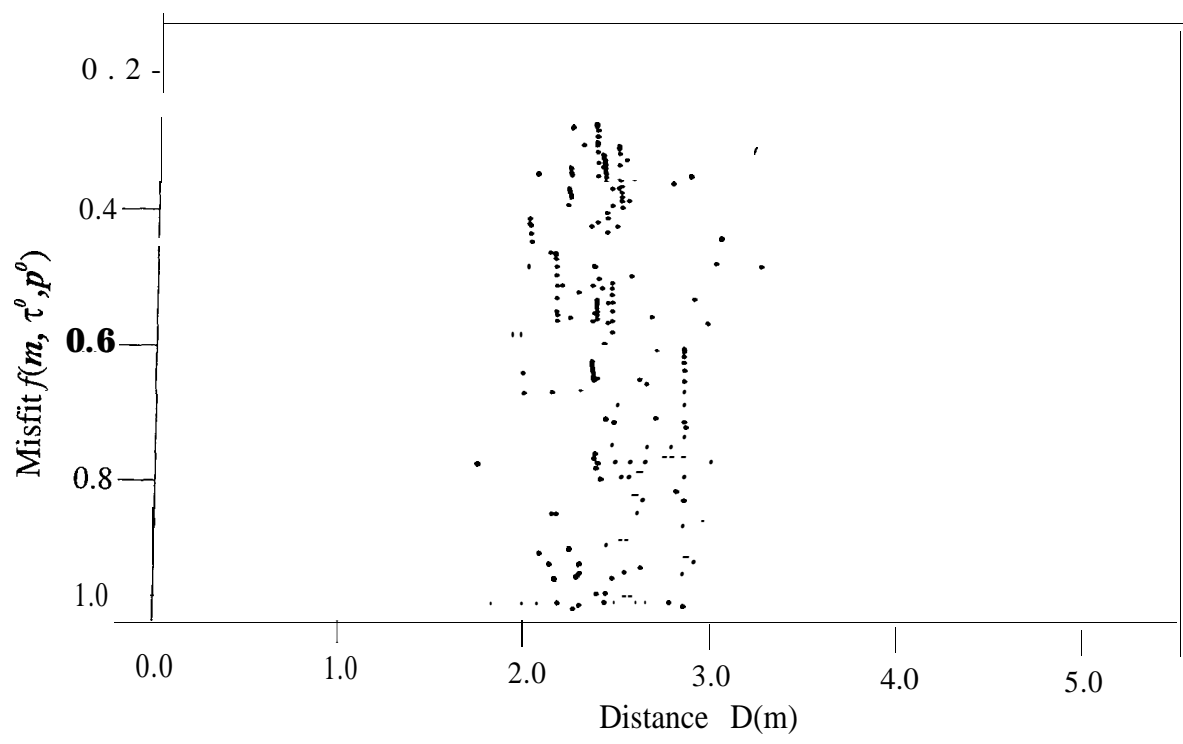

Figure 9. Distance-misfit scatter plots showing models for which the misfit is less than 1. This misfit range corresponds to models whose observations, on average, fit all the observed data within the estimated error bounds.

perpendicular to the crack strike, it can be seen that the seismic anisotropy for the dipping fractures is no longer symmetrical about the vertical axis. If we now consider two near-offset VSPs with sources located along diametrically opposite azimuths, which is approximately the case for the Peel VSP and 33-1 VSP, then the observed shear-wave birefringence is the same for vertical fracture anisotropy. However, for the subvertical fracture system, the shear-wave birefringence is greater for the VSP transmitting shear waves along the plane of fracturing than for the VSP on the opposite azimuth. This effect is observed in the two near-offset VSPs for which the time delays are larger for the 33-1 VSP compared with the Peel VSP. We propose that this technique of opposite azimuth VSP surveys may be of significant use in the discrimination and measurement of subvertical fracture systems.

\section{Resolution}

We calculate the model parameter resolution by computing misfit values along crosssections through the model space about the best solution. We achieve this by sweeping through one component of the model vector whilst keeping all other components equal to the best model parameters. The resolution for each parameter is calculated using a measure of dispersion, a, given by

$$
\sigma\left(m_{i}\right)=\left[\sum_{m_{i, \text { high }}}^{m_{i, \text { high }}}\left(m_{i, \text { best }}-m_{i}\right)^{2} L(f)_{\mathrm{I}}^{1 / 2}\right. \text {, }
$$


158 S. Horne et al.

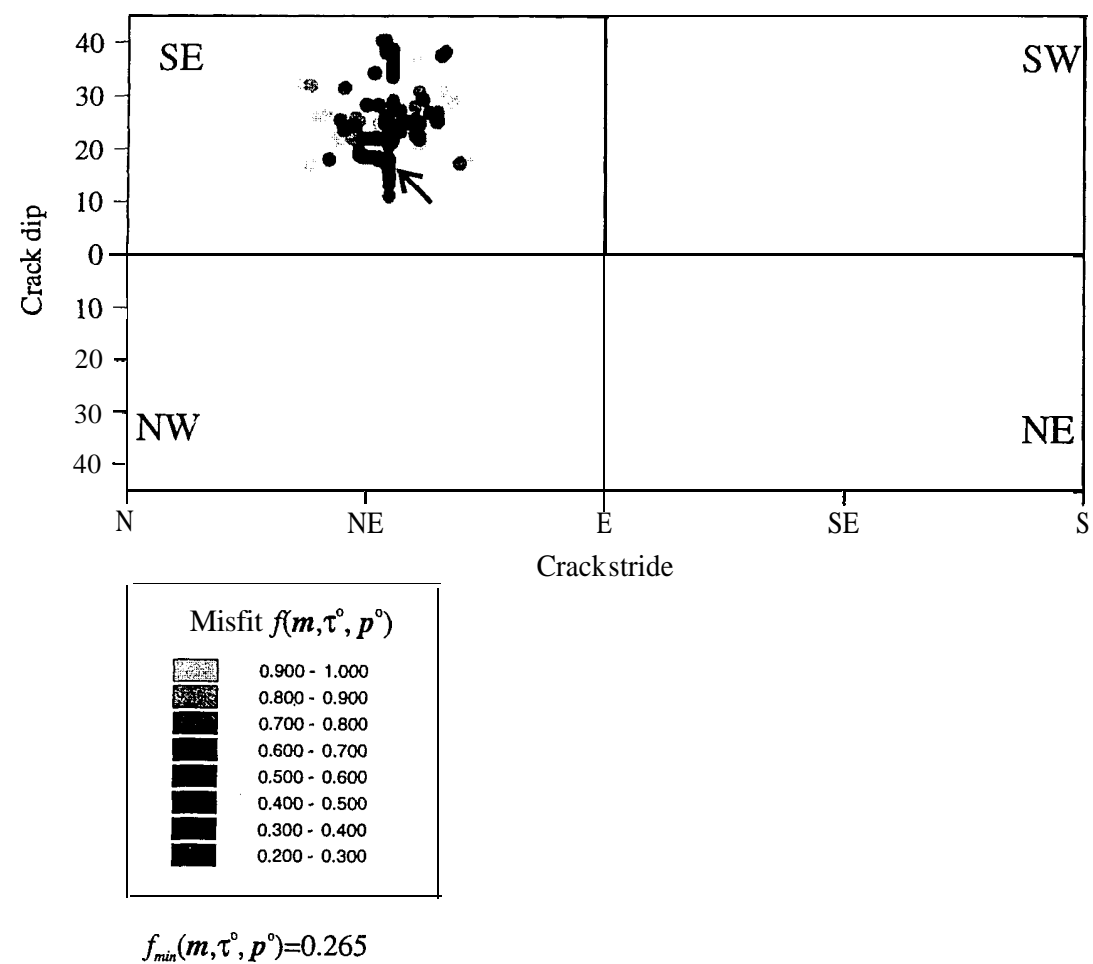

Figure 10. Scatter plot of crack strike versus crack dip for the models sampled using the GA. The dots representing the sampled models are shaded according to the solution's misfit. The direction of dip is indicated in the corner of each quadrant. The arrow indicates the best solution.

where $m_{i}$ is the ith component of the model vector m. $m_{i \text {,best }}$ is the ith component for the best model. $m_{i \text {,high }}$ and $m_{i \text {,low }}$ are the upper and lower discretization bounds. $L$ is a likelihood function defined as (Tarantola 1987)

$$
\mathbf{L}(\mathbf{x})=\mathrm{e}^{-\frac{1}{2} x},
$$

where $\mathrm{x}$ is a misfit value, such as $f, \Delta \tau$, or $\Delta p$ as defined in (2). The results of this analysis are given in Table 5 and in cross-section plots of the likelihood function for each component of the model vector (Fig. 12). The dispersion is calculated to be less than 10" for both the crack strike and the crack dip. The crack-dip cross-section plots reveal a bimodal function with two narrow peaks located at approximately opposite dips with the most significant peak located at about 20" to the south-east. This secondary maximum is located outside the window of acceptable solutions as indicated by the shaded region in the cross-section plots. If this secondary solution is omitted from the dispersion calculation, then the fracture-dip resolution reduces to 

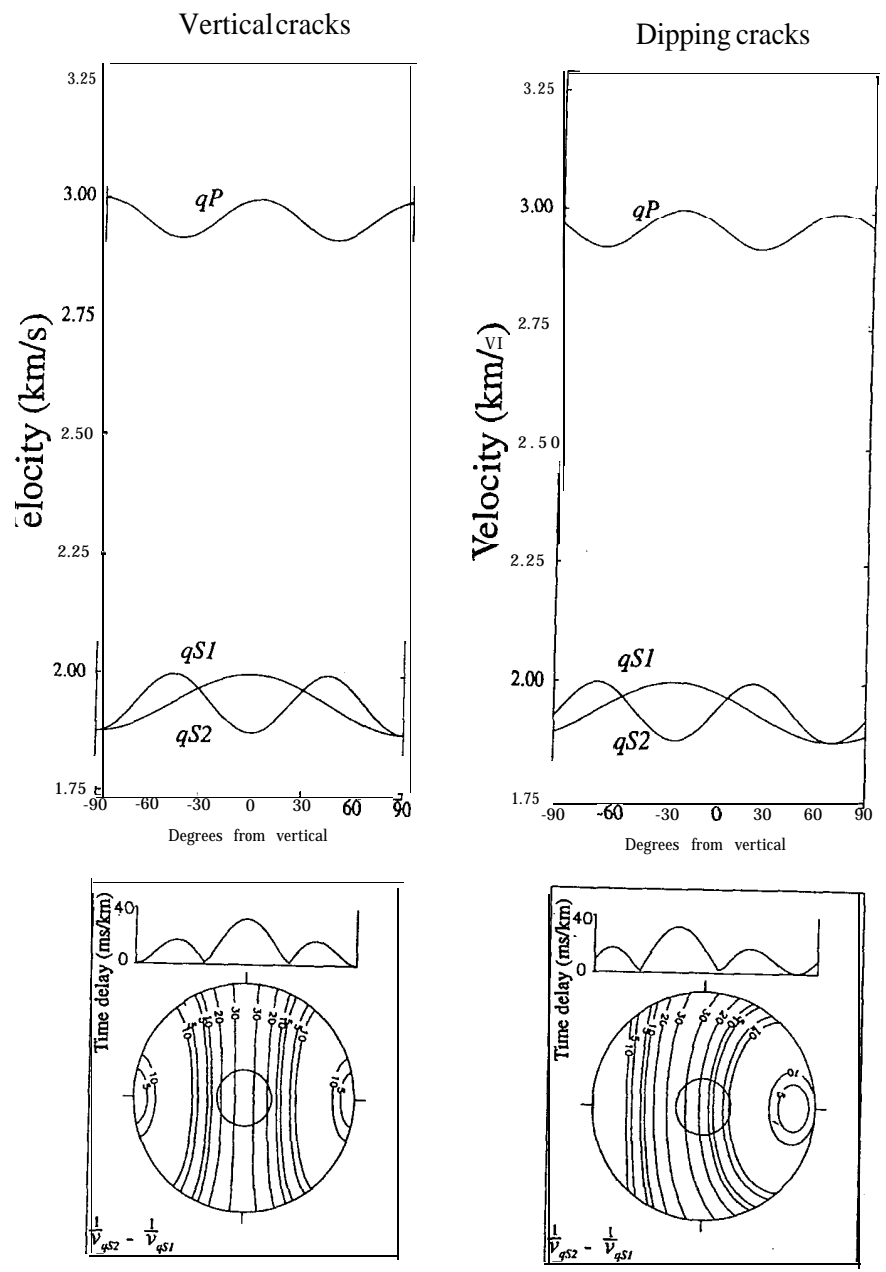

Figure 11 . A comparison of anisotropic behaviour for materials constructed from vertical (left) and subvertical (right) fractures. The subvertical cracks are rotated 20" from the vertical. The lower plots show the time-delay variations over a hemisphere of propagation directions with the inner circle indicating an equi-incident angle of $10 "$ ".

less than 1". To identify the source of this secondary solution, cross-section plots are constructed for the separate time-delay and $q S 1$ polarization misfit terms in (2) (Fig. 13). The crack dip cross-section reveals that the contribution from the $q S 1$ polarization misfit is essentially constant between dips of $\pm 25^{\circ}$. Beyond this range the likelihood function rapidly decreases to zero. This cut-off is due to the rapid changes in the predicted $q S 1$ observations which occur for these large values of dip as the line singularity is sampled. The time-delay likelihood function is bimodal with well-defined peaks at dips of 18 " to the south-east and 24 " to the north-west. The 
Table 5. Resolution estimates for the Hudson parametrization results.

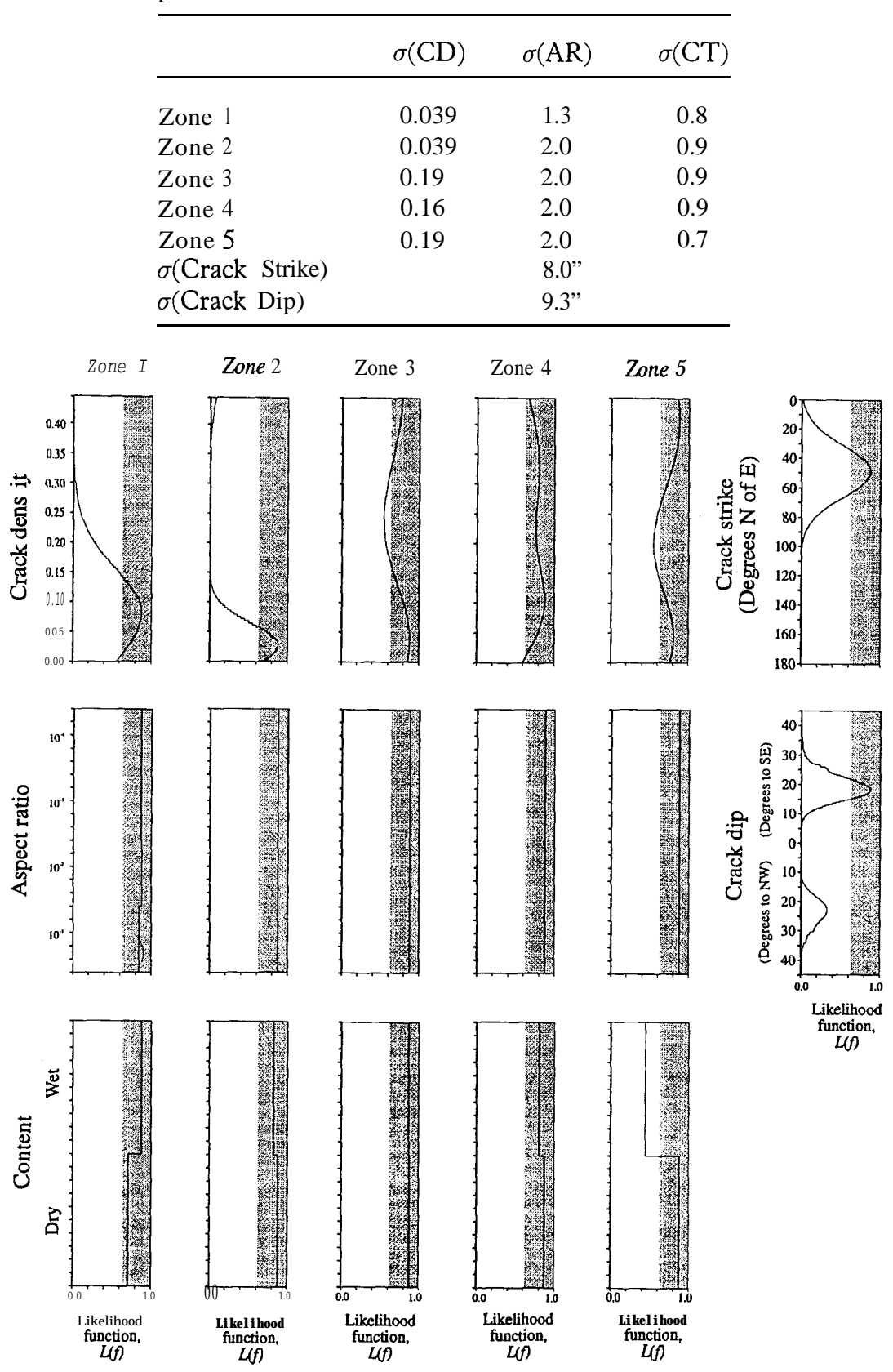

Figure 12. Likelihood cross-section plots about the best model found by the GA. The shaded regions indicate the range of acceptable solutions with misfits less than 1 .

(C) 1997 European Association of Geoscientists \& Engineers, Geophysical Prospecting, 45, 141-164 

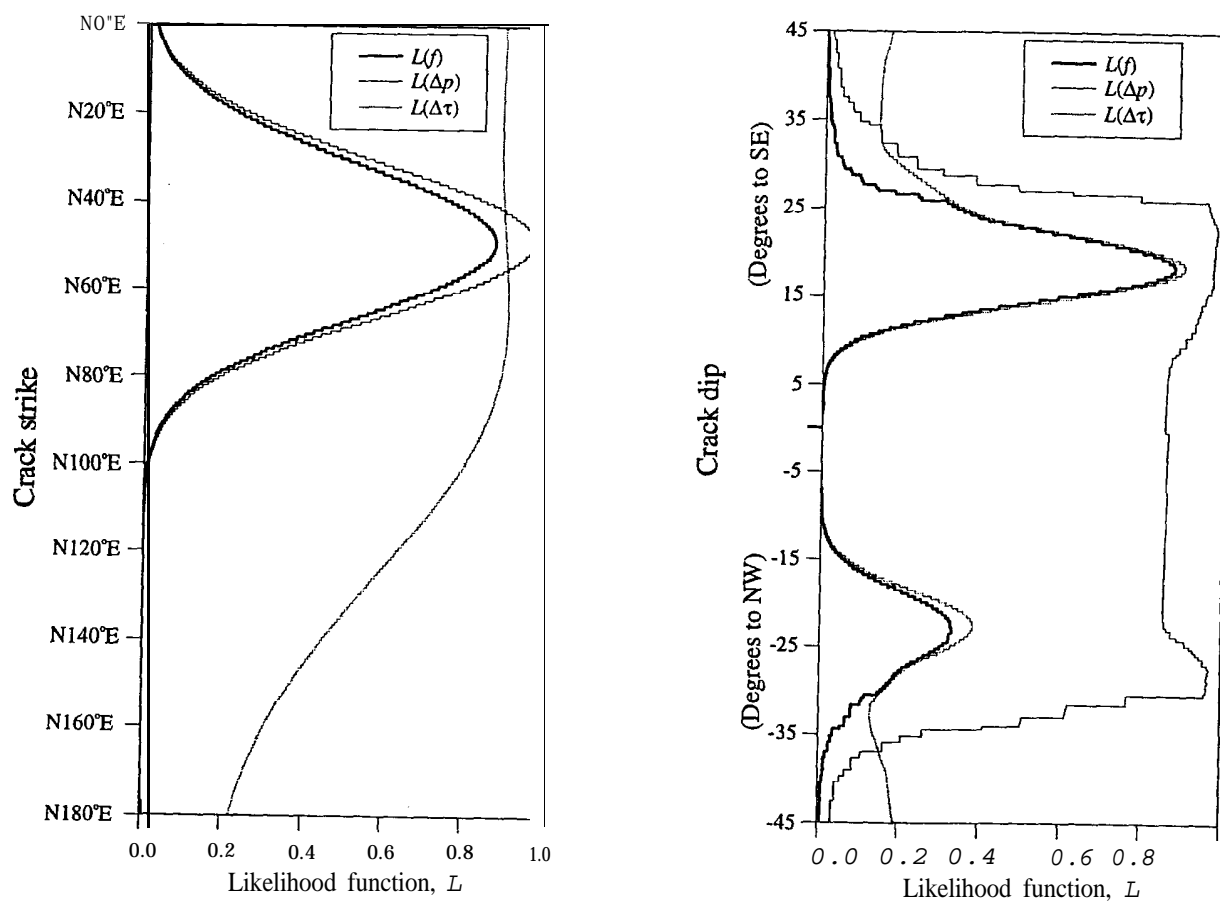

Figure 13. Cross-section plots about the best model found by the GA. The three different curves correspond to the likelihood functions for the separate time-delay and polarization misfit terms in equations (2b) and (2c) respectively.

peak located at 18 " to the south-east corresponds to the best model found by the GA. Thus it appears that the crack dip is essentially determined by the time-delay misfit term. This compares with the crack-strike parameter which is determined by the $q S 1$ polarization misfit term (Fig. 13). A possible explanation for this bi-modality is a lateral variation in the anisotropy between the two wells leading to inconsistent data. To test this supposition, we construct a noise-free synthetic data set corresponding to the two near-offset VSP geometries for the best model. Crack-orientation cross-sections are now calculated for this noise-free and consistent data set to see if the secondary maximum is eliminated (Fig. 14). The cross-sections show an almost identical form to that obtained with the observed data set. This demonstrates that lateral variations are not responsible for the bi-modality in the cross-sections. Instead, it appears that it is the acquisition geometries for these two near-offset VSPs which lead to this behaviour.

The Hudson parameters of aspect ratio and crack content are not well resolved. This low resolution is probably related to the limited sampling of angles of incidence by the near-offset VSPs. The resolution for the crack density is better than that obtained for the aspect-ratio parameters and crack content, but it is still poor. An improvement in the crack-density resolution could be achieved through an improvement in the accuracy of the time-delay measurements. This implies that the resolution of crack 
162 S. Home et al.
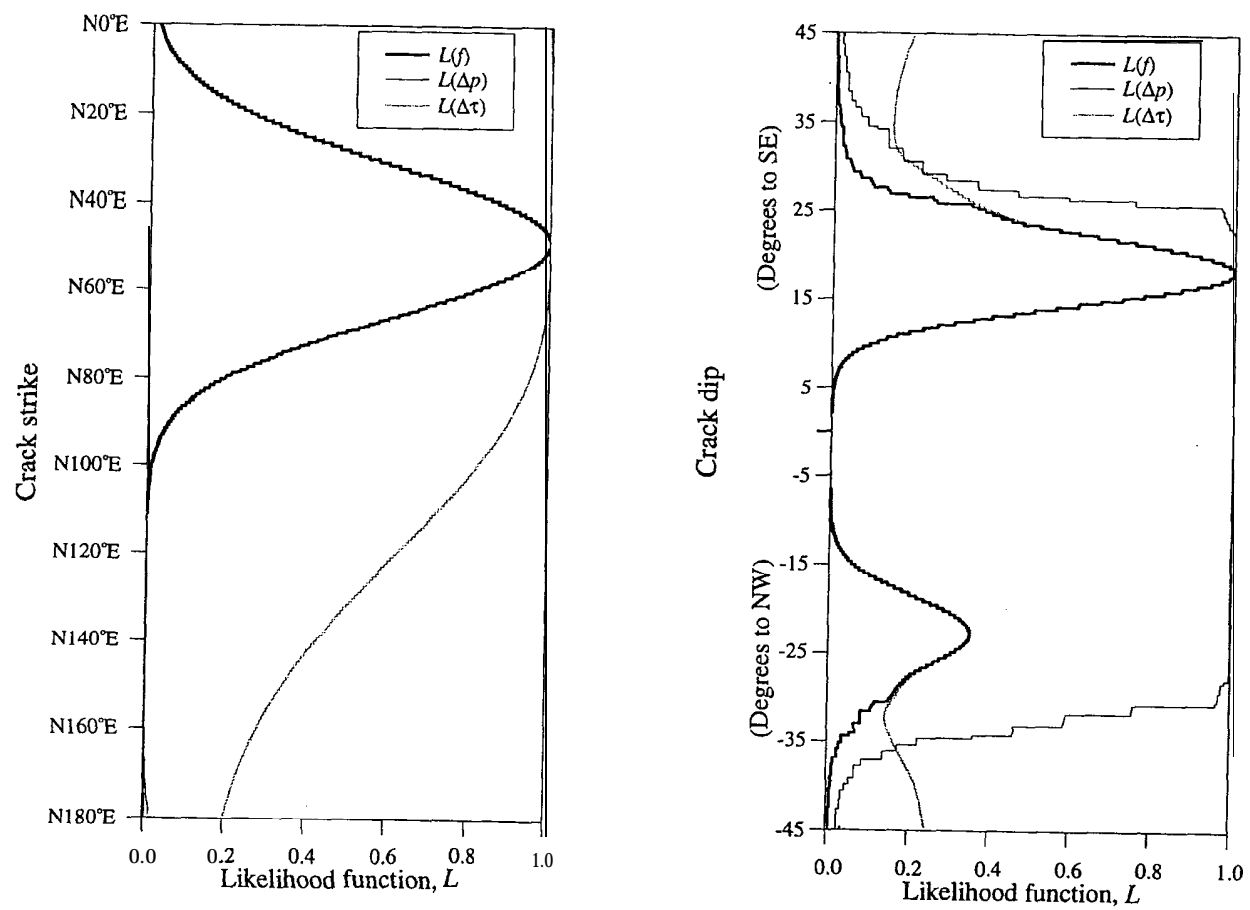

Figure 14. Likelihood cross-sections about the solution corresponding to the best model found by the GA using theoretical shear-wave splitting observations for the 33-1 and Peel geometries.

density is coupled to the time-delay resolution. Unfortunately, the error estimate of $2 \mathrm{~ms}$ is thought to be a realistic estimate which further processing will not reduce. The crack-density resolution shows a substantial decrease for the lower three zones. The reason for the relatively good resolution of the first two anisotropic zones is due to the greater number of observations in these zones. This suggests that an objective function using a normalization factor proportional to the number of observations within each zone may be more suitable for this inversion problem.

\section{Conclusions}

These inversion results support the conclusion reached by Horne and MacBeth (1994) and Liu, Crampin and Queen (1991) that the observed anisotropy at the CBTF is likely to be due to a subvertical fracture set dipping approximately 18 " to the south-east. We have also shown that the fracture dip can be obtained from measurements of shear-wave anisotropy if appropriate acquisition geometries, such as an opposite azimuth VSP, are employed. This information may be of practical importance if directional drilling is to be employed, since productivity flow rates are 
maximized for wells perpendicularly intersecting the fractures. Furthermore, an understanding of the fracture system's dip is also important with respect to the reservoir's structural behaviour, since this controls the water-cut behaviour of producing wells.

A significant correlation is observed to exist between the lithology at the CBTF and the shear-wave birefringence. Specifically, the degree of birefringence is considerably higher in the formations dominated by sandstones, suggesting that these formations may be intensely fractured. Sandstone formations, such as the Spraeberry Field, Texas, are of importance to the petroleum industry since they may often be the location of hydrocarbon reservoirs (Aguilera 1980). For these reservoirs the formation permeability anisotropy is of essential importance to production. Therefore shearwave birefringence studies may prove to be useful in these situations.

\section{Acknowledgements}

S.Horne thanks ELF GRC and the sponsors of the Edinburgh Anisotropy Project for their financial support. This work was supported by the Natural Environment Research Council, and is published with the approval of the director of the British Geological Survey.

\section{References}

Aguilera R. 1980. Naturally Fractured Reservoirs. PennWell Publishing Company, Tulsa, Oklahoma.

Ata E. and Michelena R.J. 1995. Mapping distributions of fractures in a reservoir with P-S converted waves. The Leading Edge 14, 664-676.

Červený V. 1972. Seismic rays and ray intensities in inhomogeneous anisotropic media. Geophysical Journal of the Royal Astronomical Society 29, 1-13.

Crampin S. and Lovell J.H. 1991. A decade of shear-wave splitting in the Earth's crust: what does it mean? what use can we make of it? and what should we do next? Geophysical Journal International 107, 387-408.

Davis L. 1991. Handbook of Genetic Algorithms. Van Nostrand Reinhold, New York.

Ehlig-Economides C., Ebbs D. and Meehan D.N. 1990. Factoring anisotropy into well design. Oilfield Review (October) 24-33.

Fogel D.B. and Stayton L.C. 1994. On the effectiveness of crossover in simulated evolutionary optimization. BioSystems 32, 171-182.

Fryer G.F. and Frazer L.N. 1987. Seismic waves in stratified anisotropic media -11 . Elastodynamic eigensolutions for some anisotropic systems. Geophysical Journal of the Royal Astronomical Society 91, 73-101.

Gajewski D. and Pšenčik I. 1990. Vertical seismic profile synthetics by dynamic ray tracing in laterally varying layered anisotropic structures. Journal of Geophysical Research 95, 11301-1 1315 .

Goldberg D.E. 1989. Genetic Algorithms in Search, Optimization, and Machine Learning. Addison-Wesley Pub. Co. 
Helbig K. 1994. Foundations of Anisotropy for Exploration Seismics. Elsevier Science Publishing Co.

Horne S. 1995. Applications of genetic algorithms to problems in seismic anisotropy. Ph.D thesis, University of Edinburgh.

Horne S. and MacBeth C. 1994. Inversion for seismic anisotropy using genetic algorithms. Geophysical Prospecting 42, 953-974.

Hudson J.A. 1980. Overall properties of a cracked solid. Mathematical Proceedings of the Cambridge Philosophical Society 88, 371-384.

Li Y.G., Leary P.C. and Aki K. 1990. Ray series modelling of seismic wave travel times and amplitudes in three-dimensional heterogeneous anisotropic crystalline rock: Borehole vertical seismic profiling seismograms from the Mojave Desert, California. Journal of Geophysical Research 95, 11225- 11239.

Liu E., Crampin S. and Queen J.H. 1991. Fracture detection using crosshole surveys and reverse vertical seismic profiles at the Conoco Borehole Test Facility, Oklahoma. Geophysical Journal International 107, (3), 449-463.

MacBeth C. 1991. Inversion for subsurface anisotropy using estimates of shear-wave splitting. Geophysical Journal International 107, 585-595.

MacBeth C., Zeng X., Li X.-Y. and Queen J. H. 1995. Multicomponent near-surface correction for land VSP data. Geophysical Journal International 121, 301-315.

Mueller M. 1992. Using shear waves to predict lateral variability in vertical fracture intensity. The Leading Edge 12, 29-35.

Musgrave M. J.P. 1970. Crystal Acoustics. Holden-Day, Inc.

Pereya V., Lee W.H.K. and Keller H.B. 1980. Solving two point seismic ray tracing in a heterogeneous medium, Prt I: A general adaptive finite difference method. Bulletin of the Seismological Society of America 70, 79-99.

Press W.H., Teukolsky S.A., Vetterling W.T. and Flannery B.P. 1992. Numerical Recipes in Fortran: The Art of Scientific Computing. Cambridge University Press.

Queen J.H. and Rizer W.D. 1990. An integrated study of seismic anisotropy and the natural fracture system at the Conoco Borehole Test Facility, Kay County, Oklahoma. Journal of Geophysical Research 95, 11255- 11273.

Sen M.K. and Stoffa P.L. 1992. Rapid sampling of model space using genetic algorithms: examples from seismic waveform inversion. Geophysical fournal International 108, 281-292.

Tarantola A. 1987. Inverse Problem Theory: Methods for Data Fitting and Model Parameter Estimation. Elsevier Science Publishing Co.

Zeng X. and MacBeth C. 1993. Algebraic processing techniques for estimating shear-wave splitting in zero-offset VSPs - theory. Geophysical Prospecting 41, 1033-1066.

Zoback M.L. and Zoback M. 1980. State of stress in the conterminous United States. Journal of Geophysical Research 85, 6113-6156. 